Article

\title{
Cyclic RGD Peptide Targeting Coated Nano Drug Co-Delivery System for Therapeutic Use in Age-Related Macular Degeneration Disease
}

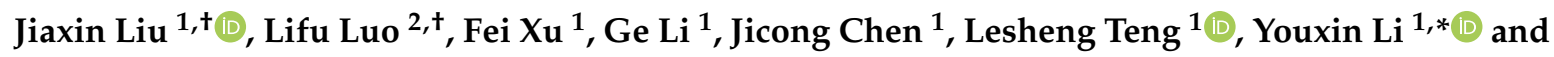 \\ Fengying Sun $1, *(\mathbb{D}$ \\ 1 School of Life Sciences, Jilin University, Changchun 130012, China; jxliu328@163.com (J.L.); \\ xufei19@mails.jlu.edu.cn (F.X.); lige18@mails.jlu.edu.cn (G.L.); chenjc20@mails.jlu.edu.cn (J.C.); \\ tenglesheng@jlu.edu.cn (L.T.) \\ 2 Department of Ophthalmology, Second Clinical College of Norman Bethune Medical Division, \\ Jilin University, Changchun 130041, China; luolf@jlu.edu.cn \\ * Correspondence: liyouxin@jlu.edu.cn (Y.L.); sunfengying@jlu.edu.cn (F.S.); \\ Tel.: +86-0431-8515-5320 (Y.L. \& F.S.) \\ + These authors contributed equally to this work.
}

Received: 30 September 2020; Accepted: 20 October 2020; Published: 23 October 2020

\begin{abstract}
Vascular endothelial growth factor (VEGF) expression increased significantly in the pathogenesis of age-related macular degeneration, which induced the formation of pathological blood vessels. Dexamethasone is an exogenous anti-angiogenic drug while bevacizumab is an endogenous anti-angiogenic drug. They both have been widely used in ophthalmology. However, independent administration is not enough to completely block the development of choroidal neovascularization $(\mathrm{CNV})$, and the number of eyes vitreous injections is limited. Reasonable combination of drugs may produce significantly better therapeutic effect than single drug treatment. The cyclic RGD (cRGD) peptide has a particularly high affinity with retinal pigment epithelial cells, where VEGF secretes from. In this study, we prepared nanoparticles of bevacizumab and dexamethasone with cRGD peptide as the target (aBev/cRGD-DPPNs). The particle size of the aBev/cRGD-DPPNs was $213.8 \pm 1.5 \mathrm{~nm}$, SEM results showed that the nano-carriers were well dispersed and spherical. The cell uptake study demonstrated the selectivity of the aBev/cRGD-DPPN to ARPE-19 with $\alpha_{V} \beta_{3}$ over expressed. The aBev/cRGD-DPPNs had a better apoptosis induction effect and an obvious inhibitory effect on migration, invasion, and capillary-like structures formation of human umbilical vein epithelial cells. The fluorescein fundus angiography study, immunohistochemistry and histopathological evaluation showed the aBev/cRGD-DPPNs greatly reduced the development of CNV on a rabbit model.
\end{abstract}

Keywords: vascular endothelial growth factor; age-related macular degeneration; anti-angiogenic drug; retinal pigment epithelial cells; nanoparticles

\section{Introduction}

According to the World Health Organization, there are about 1.3 billion people suffering from a certain degree of visual impairment and 36 million are blind worldwide [1]. Retinal diseases have become the main cause of blindness, among which age-related macular degeneration (AMD) is the leading cause. AMD is mainly divided into dry AMD and wet AMD [2,3]. Dry AMD is also known as atrophic AMD, which is clinically manifested as progressive pigment epithelial atrophy. Wet AMD is also called neovascular AMD, which is clinically manifested as active neovascularization under pigment epithelial layer. Although patients with dry AMD are much more than those with wet AMD, wet AMD has a high risk of blindness in a short period of time and more than $90 \%$ of patients with 
acute visual impairment, so wet AMD is paid more attention [4]. Choroidal neovascularization (CNV) is the main cause of visual impairment in patients with wet AMD [5].

At present, the most common drug treatment for $\mathrm{CNV}$ is anti-angiogenic drugs, which are mainly divided into endogenous anti-angiogenic drugs and exogenous anti-angiogenic drugs [6,7]. Glucocorticoids, as exogenous antiangiogenic drugs, are commonly used in clinical ophthalmology [8-10]. Dexamethasone is the most commonly used glucocorticoid and is widely used in the treatment of various diseases [11]. Studies have shown that dexamethasone can promote the regression of choroidal neovascularization by releasing local noradrenaline to constrict blood vessels, reducing or silencing the expression of vascular endothelial growth factor (VEGF), inducing the apoptosis of vascular endothelial cells and inhibiting the proliferation of vascular endothelial cells [12]. Vitreous administration is required due to its systemic side effects and the unique barrier structure of the eyeball [13,14]. However, in view of the fast metabolism of dexamethasone, the vitreous administration is not suitable for frequent operation due to pain and side effects [15-17]. Therefore, the development of suitable carriers will reduce the drug injection frequency and improve the selectivity of dexamethasone.

Endogenous antiangiogenic drugs mainly include various cytokines and cytokine antibodies or antagonists and receptor blockers [18], VEGF is the major cytokine for CNV development [19,20]. Clinically, existing anti-VEGF drugs have achieved a certain effect in the treatment of neovascular eye diseases [21,22]. Bevacizumab is the first approved anti-tumor angiogenesis drug in the USA for the treatment of metastatic colorectal cancer [23]. In recent years, bevacizumab has been widely used as "off-label" by ophthalmologists because of its efficacy in treating underlying ophthalmic diseases as well as its low cost and efficacy in the treatment of fundus diseases, which has been proved by clinical practice [24]. However, due to the complex pathogenesis of AMD, bevacizumab is not enough to completely block the development of $\mathrm{CNV}$, and the number of vitreous injections that the human eye can bear is limited. Reasonable combination of drugs may produce significantly better therapeutic effect than single drug treatment.

The pathological mechanism of CNV is the dysfunction of retinal pigment epithelial cells (RPE), which releases excessive VEGF into the choroid, and excessive VEGF can induce the formation of pathological new blood vessels of AMD $[25,26]$. The secretion of VEGF mainly comes from retinal pigment epithelial cells (RPE), so RPE are potential targets for reducing VEGF expression and preventing CNV formation in AMD. In the process of ocular neovascularization, integrin receptor $\alpha_{V} \beta_{3}$ was over expressed on the surface of RPE [27-29], and Arg-Gly-Asp (RGD) moiety containing peptide can specifically bind to integrin receptor [22,30,31]. The cyclic RGD (cRGD) peptide has a particularly high affinity with $\alpha_{V} \beta_{3}$ [32]. As a new drug delivery carrier, PLGA nanoparticles have the advantages of high biocompatibility and suitable size for cell uptake [33]. Therefore, nano-drug delivery system for co-encapsulate dexamethasone and bevacizumab modified with cRGD peptide is a promising treatment for AMD.

In the present work, we prepared the dexamethasone-loading cRGD-PEG-PLGA/PLGA/PEI nanoparticles (cRGD-DPPNs); firstly, cRGD-PEG-PLGA was added as the target to the RPE and the branched polyethylenimine (PEI) was used to regulate the potential. The adsorption of negative bevacizumab and the positive cRGD-DPPNs was to form bevacizumab-adsorbing cRGD-DPPNs (aBev/cRGD-DPPNs). Then the nanoparticles were evaluated by a series of physicochemical characterizations, in vitro stability and release behavior assay. The anti-angiogenic ability of nanoparticles to human umbilical vein epithelial cells (HUVECs) and their uptake by adult retinal pigment epithelial cells (ARPE-19) were studied. Finally, the therapeutic effect of nanoparticles on CNV was investigated by establishing CNV chinchilla rabbit model. 


\section{Results and Discussion}

\subsection{Physicochemical Characterization of the aBev/cRGD-DPPNs}

PLGA was used as the carrier material of nanoparticles, and the branched PEI was used to regulate the potential of nanoparticles, meanwhile, cRGD peptide was regarded as the target for preparing cRGD-DPPNs loaded with dexamethasone. The aBev/cRGD-DPPNs were prepared by electrostatic adsorption of the negative bevacizumab to the surface of the positive cRGD-DPPNs. Dynamic light scattering (DLS) and scanning electron microscope (SEM) images of the aBev/cRGD-DPPNs were shown in Figure 1a,b, respectively. The SEM results showed that the nanoparticles were smooth, spherical, and uniform in size. The optimal aBev/cRGD-DPPNs showed $213.8 \pm 1.5 \mathrm{~nm}$ in particle size, $0.153 \pm 0.036$ in polydispersity index (PDI), and $0.30 \pm 1.61 \mathrm{mV}$ in potential. Drug loading (DL) (\%), encapsulation efficiency (EE) (\%), and binding efficiency (BE) (\%) of the aBev/cRGD-DPPNs were $9.35 \pm 0.41 \%, 56.07 \pm 2.46 \%$, and $83.15 \pm 1.66 \%$ (Figure $1 \mathrm{c}$ ), respectively. The particles with a diameter of about $200 \mathrm{~nm}$ exhibited significantly enhanced ocular posterior segment tissues accumulation than with the other size [34].

a

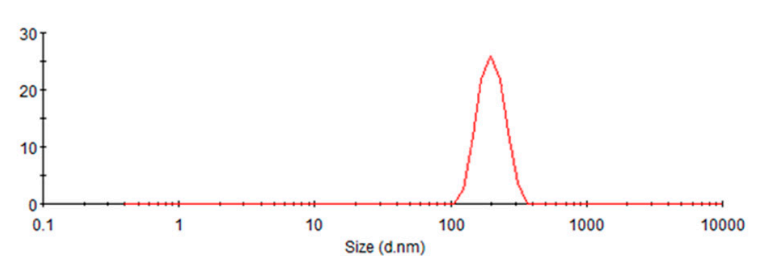

C

\begin{tabular}{ccccccc}
\hline & Size (d.nm) & PDI & $\begin{array}{c}\text { Zeta potential } \\
(\mathrm{mV})\end{array}$ & DL (\%) & EE (\%) & BE (\%) \\
\hline cRGD-DPPNs & $174.6 \pm 1.2$ & $0.243 \pm 0.086$ & $29.25 \pm 1.29$ & $10.82 \pm 0.35$ & $64.89 \pm 2.21$ & \\
$\begin{array}{c}\text { aBev/ cRGD- } \\
\text { DPPNs }\end{array}$ & $213.8 \pm 1.5$ & $0.153 \pm 0.036$ & $0.30 \pm 1.61$ & $9.35 \pm 0.41$ & $56.07 \pm 2.46$ & $83.15 \pm 1.66$ \\
\hline
\end{tabular}

b

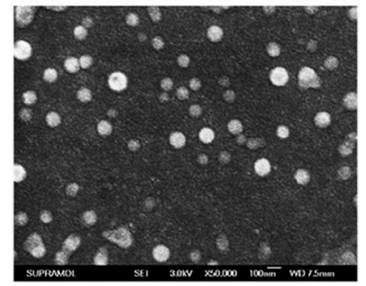

\section{.}

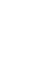


a

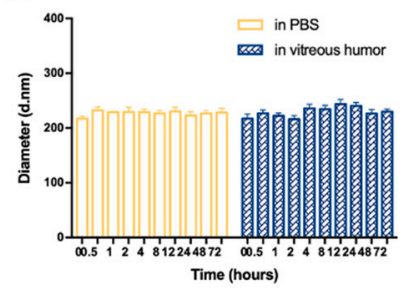

b

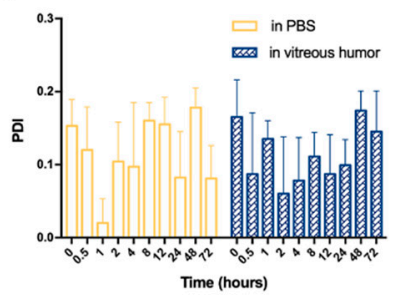

C

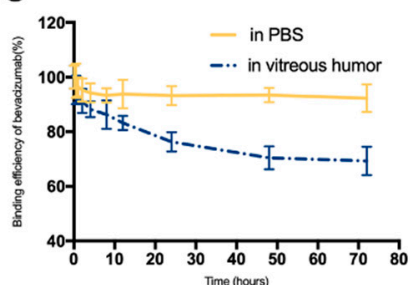

d

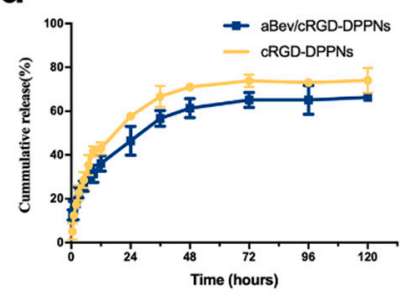

e

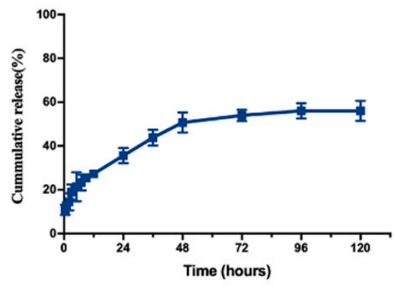

Figure 2. Stability and release behavior of the aBev/cRGD-DPPNs. (a) Stability of particle size change; (b) stability of polydispersity index (PDI) change; (c) stability of binding efficiency (BE) change; (d) in vitro release of dexamethasone from the aBev/cRGD-DPPNs and the cRGD-DPPNs; (e) in vitro release of bevacizumab from the aBev/cRGD-DPPNs. Data are expressed as mean $\pm \mathrm{SD}, n=3$.

\subsection{Sequentially Release of Dexamethasone and Bevacizumab In Vitro}

The in vitro release profiles of dexamethasone and bevacizumab from the aBev/cRGD-DPPNs and the cRGD-DPPNs in release medium were investigated. The release result of dexamethasone was shown in Figure $2 \mathrm{~d}$. At $12 \mathrm{~h}$, the burst release of dexamethasone from the aBev/cRGD-DPPNs and the cRGD-DPPNs was $36.16 \pm 3.52 \%$ and $43.05 \pm 2.72 \%$, respectively. The cumulative release from the aBev/cRGD-DPPNs and the cRGD-DPPNs was $66.32 \pm 1.38 \%$ and $74.01 \pm 5.72 \%$ at $120 \mathrm{~h}$, respectively.

The result of bevacizumab release was shown in Figure 2e. At $12 \mathrm{~h}$, the burst release of bevacizumab from the aBev/cRGD-DPPNs was $27.26 \pm 1.54 \%$. At $12 \mathrm{~h}$, the burst release of bevacizumab from the aBev/cRGD-DPPNs was $56.20 \pm 4.59 \%$. The subsequent release of dexamethasone and bevacizumab from the aBev/cRGD-DPPNs could expose drugs to the CNV area with a long-term effective drug concentration.

\subsection{Cellular Uptake of the aBev/cRGD-DPPNs}

Confocal laser scanning microscopy (CLSM) was used to observe the drug distribution in ARPE-19 cells and 293T cells after internalization of nanoparticles. Compared with the overexpression of integrin $\alpha_{\mathrm{v}} \beta_{3}$ in ARPE-19 cells, the surface of 293T cells had an extremely low expression of integrin $\alpha_{v} \beta_{3}$ [35]. We used double fluorescent labeled nanoparticles to investigate the degree of cell internalization of nanoparticles. The results are shown in Figure $3 a, b$. The blue fluorescence represents DAPI-labeled cell nucleus. The red fluorescence was the core of the DPPNs, the aBev/DPPNs or the aBev/cRGD-DPPNs labeled by hydrophobic Rhod B. FITC-labeled bevacizumab shell was with green fluorescence. In ARPE-19 cells, it can be seen from the merged image that the nucleus of the aBev/cRGD-DPPNs group was surrounded by red and green fluorescence, which proves that the aBev/cRGD-DPPNs were internalized into the cell nearly at the same time, and bevacizumab did not dissociate during the process of cell internalization. However, the fluorescence intensity of the DPPNs and the aBev/DPPNs in ARPE-19 cells was very weak, which indicates that the aBev/cRGD-DPPNs has higher cellular internalization efficiency, and the aBev/cRGD-DPPNs can target ARPE-19 cells more effectively. However, no obvious red and green fluorescence was observed in 293T cells, indicating that the aBev/cRGD-DPPNs have a targeting selectivity for the ARPE-19 cells over expressing integrin $\alpha_{\mathrm{v}} \beta_{3}$. 
a

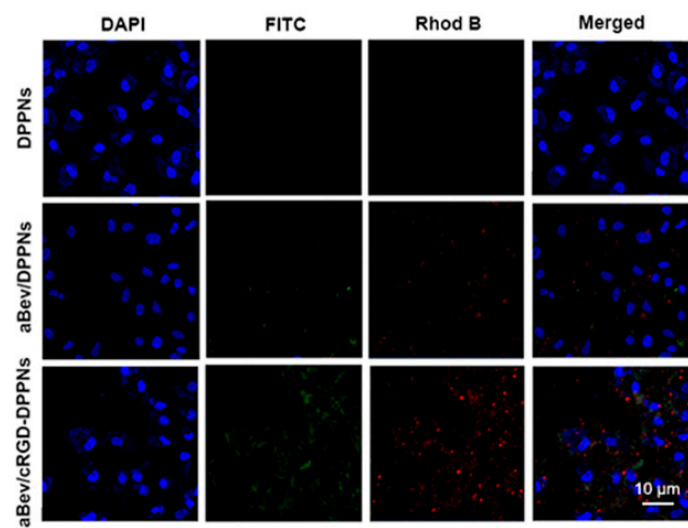

b

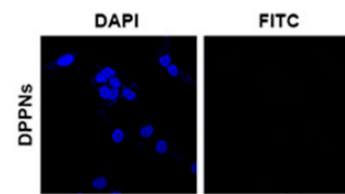

Rhod B
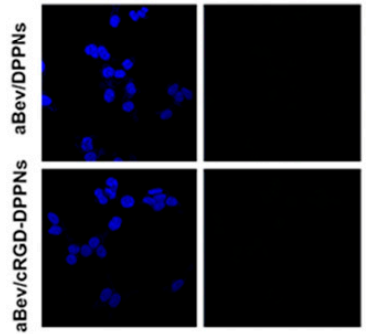

C

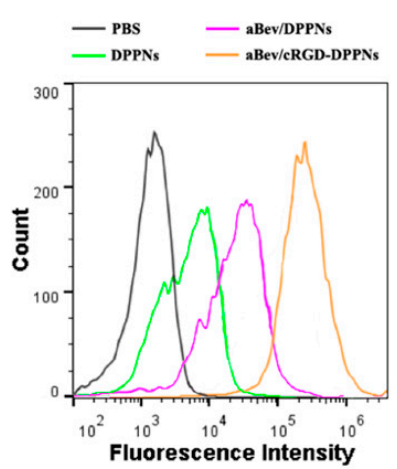

d
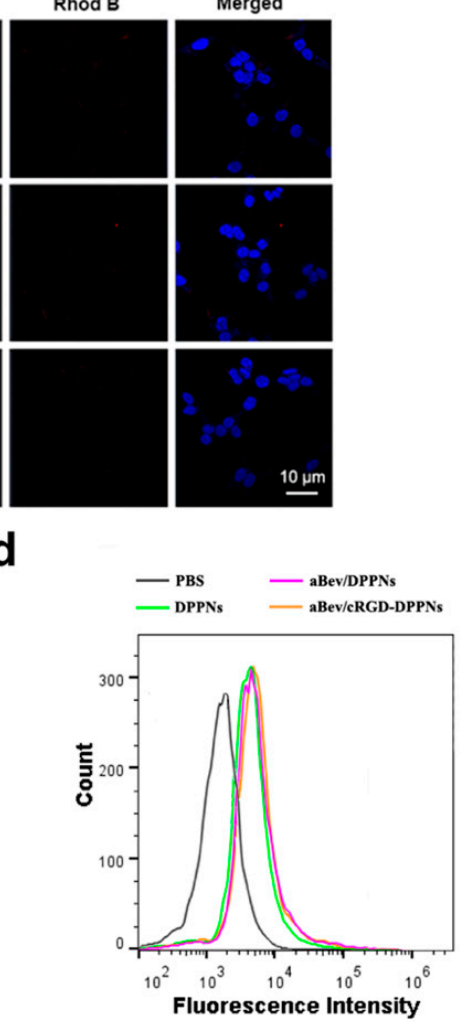

Figure 3. Cellular uptake of the aBev/cRGD-DPPNs. (a) Uptake of nanoparticles in ARPE-19 cells by Confocal laser scanning microscopy (CLSM); (b) uptake of nanoparticles in 293T cells by CLSM; (c) uptake of nanoparticles in ARPE-19 cells by Flow cytometry (FCM); (d) uptake of nanoparticles in 293T cells by FCM.

Flow cytometry (FCM) was used to investigate the internalization efficiency of nanoparticles in ARPE-19 cells and 293T cells. Since the two fluorescence distributions measured by CLSM were similar, only Rhod B was used to mark the nanoparticles in this experiment. As seen in Figure 3c, the curve of the aBev/cRGD-DPPNs shifted significantly to the right, and the degree of cell internalization was positively correlated with the fluorescence intensity of Rhod B, indicating that the aBev/cRGD-DPPNs were absorbed by ARPE-19 cells more than the DPPNs even the aBev/DPPNs. However, there was no significant difference in Rhod B fluorescence intensity of the DPPNs, the aBev/DPPNs and the aBev/cRGD-DPPNs in 293T cells (Figure 3d), which showed that the enhanced cell internalization efficiency of the aBev/cRGD-DPPNs in ARPE-19 cells was attributed to the targeted delivery of nanoparticles. The enhanced association of aBev/cRGD-DPPNs compared to unmodified aBev/DPPNs was contributed by cRGD. This result is consistent with those of CLSM. 


\subsection{Apoptosis, Wound Healing, Transwell Invasion, and Tube Formation Assay of HUVECs}

Endothelial cell apoptosis plays a critical role in the angiogenic process. FITC annexin V/PI apoptosis detection kit was used to evaluate the induction of apoptosis by nanoparticles. As shown in Figure $4 \mathrm{a}$, there was almost no apoptosis in the control group. The apoptosis percent of the DPPNs, the aBev/DPPNs, and the aBev/cRGD-DPPNs was 33.04 $\pm 3.53 \%, 69.53 \pm 2.87 \%$, and $77.63 \pm 3.82 \%$, respectively. The results showed that the aBev/cRGD-DPPNs can significantly induce apoptosis of HUVEC cells $(p<0.001)$.

a

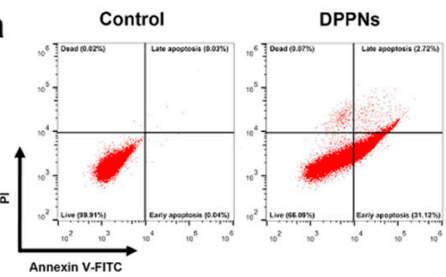

b
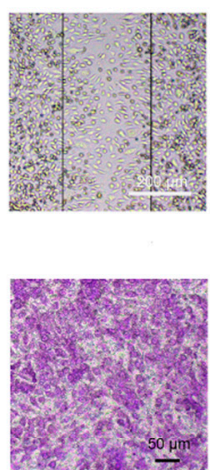

d

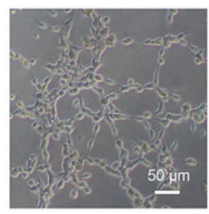

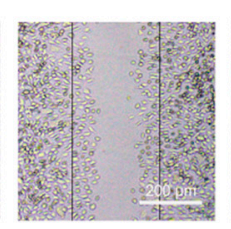
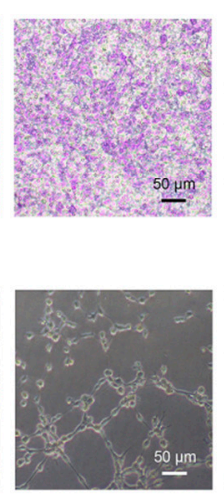
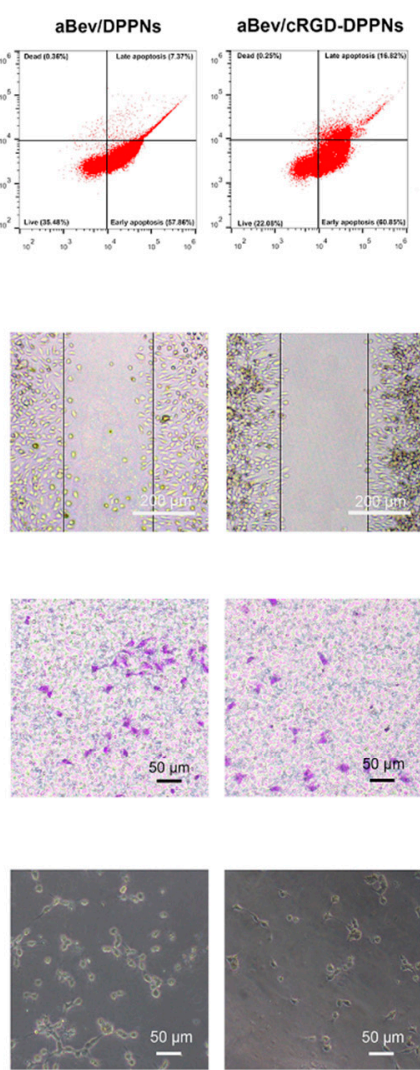
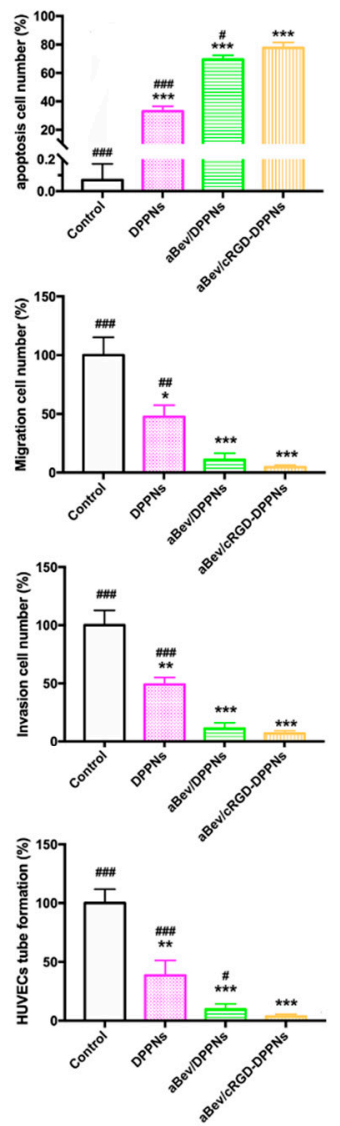

Figure 4. The aBev/cRGD-DPPNs induced apoptosis and inhibited migration, invasion, and tube formation of HUVECs. (a) The aBev/cRGD-DPPNs induced apoptosis of HUVECs; (b) the aBev/cRGD-DPPNs inhibited HUVECs migration in wound healing assay; (c) the aBev/cRGD-DPPNs inhibited HUVECs invasion in Transwell assay; (d) the aBev/cRGD-DPPNs inhibited tube formation of HUVECs. Data are expressed as mean $\pm \mathrm{SD}, n=6 .{ }^{*} p<0.05,{ }^{* *} p<0.01$, ${ }^{* * *} p<0.001$ vs. the control; \# $p<0.05$, \#\# $p<0.01$, \#\#\# $p<0.001$ vs. the aBev/cRGD-DPPNs.

Endothelial cell migration and invasion are crucial steps in angiogenesis. The inhibition of the nanoparticles on endothelial cell migration and endothelial cell invasion was evaluated by wound healing assay and Transwell assay. We found that all the DPPNs, the aBev/DPPNs, and the aBev/cRGD-DPPNs inhibited migration of HUVECs (Figure $4 b$ ), and the aBev/cRGD-DPPNs showed a smaller migration effect than the DPPNs and the aBev/DPPNs $(47.68 \pm 9.88 \%$ vs. $4.73 \pm 1.57 \%, p<0.01 ; 10.95 \pm 5.62 \%)$. In Figure $4 c$, the aBev/cRGD-DPPNs showed a smaller invasion effect than the DPPNs and the aBev/DPPNs $(49.17 \pm 5.98 \%$ vs. $6.81 \pm 2.36 \%, p<0.001 ; 11.23 \pm 4.80 \%)$.

During the late stage of angiogenesis, after the endothelial cells have finished migrating and invading, the endothelial cells will further arrange into tube-like structures. We further evaluated the inhibitory effect of nanoparticles on the ability of tube formation on matrix gel. The results in Figure $4 \mathrm{~d}$ showed the aBev/cRGD-DPPNs induced a stronger HUVECs tube formation inhibitory effect than the 
DPPNs $(p<0.001)$ and the aBev/DPPNs $(p<0.05)$. These results illustrated that the aBev/cRGD-DPPNs had significant anti-angiogenic effects on HUVECs.

\subsection{In Vivo CNV Inhibition Study}

The CNV growth inhibition study was studied by fluorescein fundus angiography (FFA). FFA was conducted 4 weeks after administration. The contrast agent of $10 \%$ fluorescein sodium $(0.2 \mathrm{~mL})$ and $0.9 \%$ saline $(1 \mathrm{~mL})$ were injected through the peripheral vein of the ear. FFA photo was recorded with high performance digital imaging system (Heidelberg HRA-II, Germany).

FFA was performed to examined CNV development. Four weeks after intravitreal injection of the drugs, the laser lesions of fluorescent leakage were evaluated by FFA (Figure 5a). Large and diffuse areas of leakage were observed in the eyes of thenon-treated CNV group. The intensity of fluorescent leakage intensity in the DPPNs, the aBev/DPPNs, and the aBev/cRGD-DPPNs group was $62.53 \pm 9.66 \%$, $27.17 \pm 8.82 \%$, and $12.58 \pm 3.12 \%$, respectively. The inhibitory effects of the aBev/cRGD-DPPNs on the area of CNV leakage were statistically significant $(p<0.001)$.

a

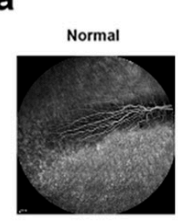

b

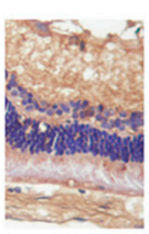

C

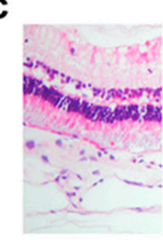

Control
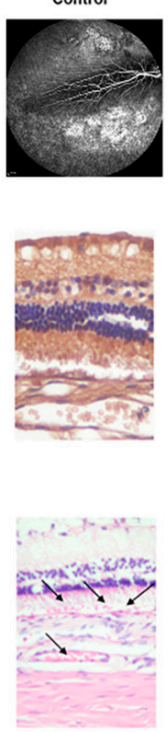

DPPNs
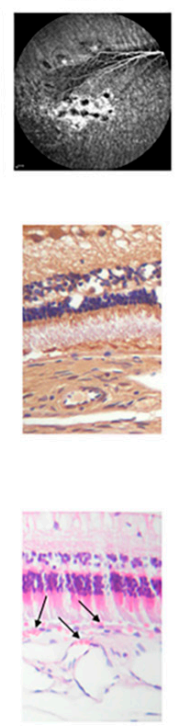

aBev/DPPNs
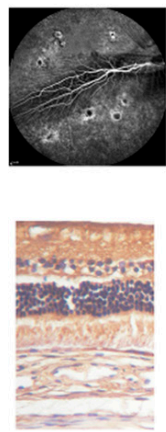

2

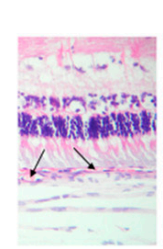

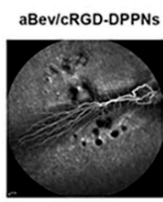

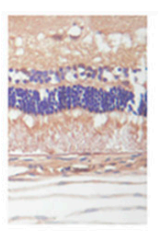

d

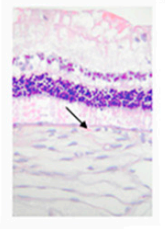

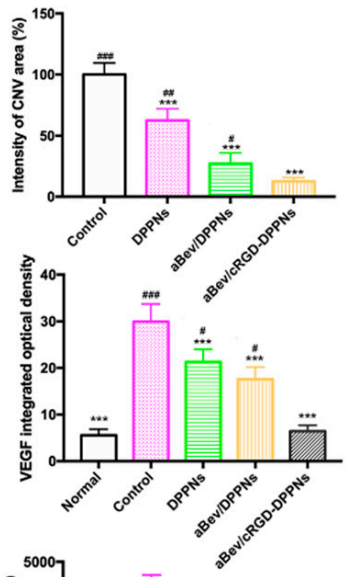

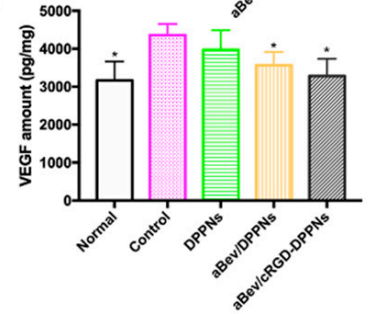

Figure 5. The aBev/cRGD-DPPNs inhibited choroidal neovascularization (CNV) development in vivo. (a) The aBev/cRGD-DPPNs decreased the area of CNV leakage by fluorescein fundus angiography (FFA); (b) immunohistochemical results of the aBev/cRGD-DPPNs treated CNV rabbits; (c) histopathology analysis of the aBev/cRGD-DPPNs treated CNV rabbits; (d) VEGF amount in the aBev/cRGD-DPPNs treated CNV rabbits. Data are expressed as mean $\pm \mathrm{SD}, n=3$. ${ }^{*} p<0.05$, ${ }^{* *} p<0.001$ vs. the control; $\# p<0.05, \# \# p<0.01$, \#\#\# $p<0.001$ the aBev/cRGD-DPPNs.

Immunohistochemistry results of VEGF protein expressed in the retina-choroid appeared brownish-yellow staining in the slices, and the darker of brownish. IOD values of the positive staining area were positively correlated with VEGF expression, as shown in Figure 5b. The IOD value of the normal group, the control group, the DPPNs, the aBev/DPPNs, and the aBev/cRGD-DPPNs group was $5.58 \pm 1.30,29.91 \pm 3.79,21.31 \pm 2.72,17.59 \pm 2.58$, and $6.46 \pm 1.24$, respectively. The expression of VEGF in the control group was significantly higher than that in the normal group $(p<0.001)$. The aBev/cRGD-DPPNs showed the most significant inhibitory effect on VEGF $(p<0.001)$.

HE staining results of the retina-choroid tissue are shown in Figure $5 \mathrm{c}$. The structure of normal tissue was complete and clear. In the CNV control group, there were obvious nuclear condensation in the inner and outer nuclear layers, cell atrophy in the photoreceptor layer, scar formation in the RPE 
cell layer toward the retina, and obvious growth of CNV (black arrows). After administration of the DPPNs, the aBev/DPPNs or the aBev/cRGD-DPPNs, the typical CNV structure was reduced, and the CNV lesions structure showed a significantly decreased in the aBev/cRGD-DPPNs group.

Previous studies have shown that VEGF is mainly expressed during the embryonic stage, plays an important role in the formation and development of blood vessels. Furthermore, VEGF can be expressed in the process of angiogenesis. Therefore, the expression level of VEGF protein in RPE-choroidal complex was quantitatively analyzed by ELISA, as shown in Figure 5d. The content of VEGF in RPE choroidal complex of CNV rabbit model can be effectively reduced by intravitreal administration of the aBev/cRGD-DPPNs $(p<0.05)$.

\section{Materials and Methods}

\subsection{Materials}

Dexamethasone and FITC were purchased from Yuanye Biotechnology (Shanghai, China). Bevacizumab (molecular weight (MW) $149 \mathrm{kDa}$ ) was donated by Luye Pharmaceutic (Yantai, China). PLGA 503H was purchased from Evonic Industries (Birmingham, AL, Germany). cRGD-PEG-PLGA was purchased from Xi'an Ruixi biotechnology ( $\mathrm{Xi}^{\prime}$ an, China). Micro bicinchoninic acid (BCA) protein assay kits were purchased from Thermo Scientific (Waltham, MA, USA). PEI (MW $25 \mathrm{kDa}$, branched) and polyvinyl alcohol (PVA) (MW 13-23 kDa) were purchased from Sigma-Aldrich (St Louis, MO, USA). Rhod B was purchased from Sinopharm chemical reagents (Beijing, China). Acetonitrile was purchased from Thermo Fisher Scientific (Waltham, MA, USA). Rabbit VEGF enzyme-linked immunosorbent assay (ELISA) kits were obtained from Elabscience Biotechnology (Wuhan, China). All other chemicals used were of analytical grade and used without further purification.

\subsection{Preparation of the aBev/cRGD-DPPNs}

The cRGD-DPPNs containing PLGA and cRGD-PEG-PLGA with a proportion of 9:1 (w/w) were prepared by an emulsion solvent volatilization method. Briefly, PLGA (22.5 mg) together with cRGD-PEG-PLGA $(2.5 \mathrm{mg}$ ) were dissolved in $1.5 \mathrm{~mL}$ chloroform and mixed with dexamethasone $(6 \mathrm{mg})$ dissolved in $1 \mathrm{~mL}$ acetone. The above mixed solution was added to $1 \%$ PVA ( $w / v)$ aqueous solution containing PEI with positive charge dropwise and was subject to phacoemulsification. The cRGD-DPPNs were formed and dried with a magnetic stirrer at $25{ }^{\circ} \mathrm{C}$ over night to remove the residual solvent completely. Meanwhile, the preparation of DPPNs was the same as that of cRGD-DPPNs except that the cRGD-PEG-PLGA was replaced by an equal amount of PLGA.

The aBev/cRGD-DPPNs were prepared by the adsorption method based on the method reported before [36]. A certain mass of bevacizumab and the cRGD-DPPNs were co-dispersed in PBS (pH 8.0) for $2 \mathrm{~h}$ at $25^{\circ} \mathrm{C}$. Ultrafiltration tubes (MWCO $300 \mathrm{kDa}$ ) were used to remove unconnected bevacizumab and salt ions from the solution. Freeze-dried after $-20^{\circ} \mathrm{C}$ storage in the dark reserve. The final products were lyophilized.

The bevacizumab-adsorbing dexamethasone-loading PLGA/PEI nanoparticles (aBev/DPPNs) were fabricated for comparison.

\subsection{Physicochemical Characterization of the aBez/cRGD-DPPNs}

The size, distribution, and potential of the nanoparticles were measured by ZS90 dynamic light scattering (DLS) (Malvern, UK).

Then the morphological characteristics of the aBev/cRGD-DPPNs were observed by scanning electron microscope (SEM) (JXA-840, JEOL, Tokyo, Japan) sample platform. The aBev/cRGD-DPPNs were diluted with deionized water to $1 \mathrm{mg} / \mathrm{mL}$, respectively. The nanoparticles suspension was dropped on the clean silicon wafer, and the surface liquid was dried overnight at room temperature. The silicon wafer was fixed and sprayed with gold in a vacuum for $20 \mathrm{~s}$. The test voltage was set to $3 \mathrm{kV}$. 
The dexamethasone encapsulation efficiency (EE) into the aBev/cRGD-DPPNs were detected using HPLC (Waters, Milford, MA, USA) at $240 \mathrm{~nm}$. The mobile phase composed of acetonitrile and water at a ratio of 60:40 (v/v) was loaded onto an Agilent XDB-C18 column (Agilent Technologies, Lexington, MA, USA, $4.6 \mathrm{~mm} \times 250 \mathrm{~mm}, 5 \mu \mathrm{m})$. Briefly, the content of dexamethasone was evaluated via dissolving the aBev/cRGD-DPPNs in $1 \mathrm{~mL}$ of acetonitrile and subsequently diluted to $5 \mathrm{~mL}$ with the mobile phase. The drug loading (DL) was calculated as the ratio of the amount of dexamethasone encapsulated in the nanoparticles to the total amount of the nanoparticles. The EE was calculated as the ratio of the actual dexamethasone loading to the theoretical dexamethasone loading. The bevacizumab binding efficiency (BE) in the aBev/cRGD-DPPNs was examined via collecting the solution removed from ultrafiltration tubes centrifugation and quantitively analyzed by a Micro BCA protein assay. The BE was calculated as the ratio of the amount of total bevacizumab subtract free bevacizumab to the total amount of the nanoparticles.

\subsection{Stability of the aBev/cRGD-DPPNs}

To investigate the stability of the aBev/cRGD-DPPNs under in vitro physiological conditions, the aBev/cRGD-DPPNs were suspended in PBS of pH 7.4 and fresh rabbit eye vitreous, respectively, and then stored at $37^{\circ} \mathrm{C}$. The particle size, PDI, and BE were measured at predetermined time with the methods mentioned above.

\subsection{Sequentially Release of Dexamethasone and Bevacizumab In Vitro}

The nanoparticles were suspended in $1 \mathrm{~mL}$ release medium of PBS containing $0.5 \%$ Tween- 80 of $\mathrm{pH}$ 7.4. Then the dialysis bag (MWCO $8 \mathrm{kDa}$ ) containing mixed suspension was put into the centrifuge tube containing $50 \mathrm{~mL}$ same release medium in a shaker at $37^{\circ} \mathrm{C}$ with $110 \mathrm{rpm}$. At pre-specified time intervals, $2 \mathrm{~mL}$ of releasing medium was taken out, and the equal volume fresh releasing medium was added. The dexamethasone amount was determined by HPLC and the bevacizumab amount was determined by the Micro BCA protein assay.

\subsection{Cellular Uptake of the aBev/cRGD-DPPNs}

The cellular uptake of nanoparticles was performed using fluorescently labeled nanoparticles and measured by confocal laser scanning microscopy (CLSM) and flow cytometry (FCM) analysis. Dexamethasone was replaced by fluorescent dye Rhod B, and Bev was labeled with FITC. Briefly, Bev and FITC had the proportion of 1:7 $(w / w)$. The FITC solution was added to the Bev solution dropwise and the reaction was stopped by $5 \mathrm{M} \mathrm{NH}_{4} \mathrm{Cl}$. The reaction solution was dialyzed overnight to remove unreacted FITC. The $\alpha_{\mathrm{V}} \beta_{3}$-positive ARPE- 19 cells were incubated at $37^{\circ} \mathrm{C}$ in a $5 \% \mathrm{CO}_{2}$ in $\mathrm{RPMI}$ 1640 (Hyclone, Logan, UT, USA) with 10\% FBS (Procell, Wuhan, China) and 1\% penicillin/streptomycin (Gibco, Grand Island, NE, USA). The $\alpha_{V} \beta_{3}$-negative Human embryonic kidney cells (293T) were incubated at $37^{\circ} \mathrm{C}$ in a $5 \% \mathrm{CO}_{2}$ in DMEM (Gibco) with $10 \% \mathrm{FBS}$ and $1 \%$ penicillin/streptomycin.

ARPE-19 cells and 293T cells in the log growth stage were seeded into 12-well plates containing climbing tablets and cultured for $24 \mathrm{~h}$ making the cells grow to $75 \%$. The original cell culture medium was discarded, and the fluorescently-labeled the DPPNs, the aBev/DPPNs, the aBev/cRGD-DPPNs were added to continue the dark culture for $2 \mathrm{~h}$. Then, the cells were rinsed gently 3 times with PBS, and fixed with Verhoeff's solution for $15 \mathrm{~min}$. Then, the cells were treated with Triton X-100 for $5 \mathrm{~min}$ to increase cell permeability, stained cellular nuclei with DAPI dye solution, and imaged under CLSM (LSM710, Carl Zeiss, Berlin, Germany).

To quantify the uptake of nanoparticles by ARPE-19 cells and 293T cells, the cells in the log growth stage were seeded into 6-well plates and placed in a $5 \% \mathrm{CO}_{2} 37{ }^{\circ} \mathrm{C}$ incubator for $24 \mathrm{~h}$. The original cell culture medium was discarded and Rho B-labeled the DPPNs, the aBev/DPPNs or the aBev/cRGD-DPPNs were added. The cells were incubated for an additional $4 \mathrm{~h}$ and rinsed gently with PBS 3 times, $200 \mu \mathrm{L}$ of trypsin cells were added to each well, and the cells were re-suspended in the 
pre-cooled PBS, and the collected cells were examined by FCM (Beckman Coulter, Brea, CA, USA) to determine the cellular uptake efficiency of the nanoparticles.

\subsection{Apoptosis, Wound Healing, Transwell Invasion, and Tube Formation Assay of HUVECs}

HUVECs were incubated at $37^{\circ} \mathrm{C}$ in a $5 \% \mathrm{CO}_{2}$ in Medium 199 (Gibco) supplemented with $10 \%$ FBS and $1 \%$ penicillin/streptomycin. The DPPNs, the aBev/DPPNs and the aBev/cRGD-DPPNs were suspended in serum-free culture medium for cell cultures.

HUVEC s were collected and cultured in 6-well plates. After growing to $80 \%$, the starved cells were treated with drugs (the DPPNs, the aBev/DPPNs or the aBev/cRGD-DPPNs). After $24 \mathrm{~h}$ of treatment, 50,000 100,000 cells were taken, the supernatant was centrifuged and Annexin V-FITC and PI staining solution were added and gently mixed. Following incubation for $15 \mathrm{~min}$, the cells were washed and analyzed by FCM.

The wound healing assay was performed as described previously [37]. HUVECs were cultured into 12-well plates. After the cells grew full, the monolayer HUVECs were scratched with $20 \mu \mathrm{L}$ straws tips. Serum-free medium containing the DPPNs, the aBev/DPPNs or the aBev/cRGD-DPPNs was added respectively, and the pure medium was used as control. The migrated cells were quantified (CKX41, Olympus, Tokyo, Japan) by artificial counting after $24 \mathrm{~h}$. The inhibition rate was calculated based on $100 \%$ of HUVECs treated with the medium.

The inhibition of the nanoparticles on cell invasion was evaluated by Transwell invasion assay. The Transwell chambers ( $8 \mu \mathrm{m}$ microporous) were placed in 24 -well plates, and the top chamber was added with $50 \mu \mathrm{L}$ matrix gel on the ice, and kept at $37^{\circ} \mathrm{C}$ for $10 \mathrm{~min}$. In the top chamber, $200 \mu \mathrm{L}$ of $4 \times 10^{4}$ HUVECs in the pure medium containing the DPPNs, the aBev/DPPNs, or the aBev/cRGD-DPPNs were added. The plate wells with the chambers were filled with medium containing VEGF165 medium. HUVECs were allowed to migrate for $24 \mathrm{~h}$. The cells were fixed with $100 \%$ methanol, washed with PBS for three times, then stained with $0.05 \%$ crystal violet. Then the invasive cells were counted and quantified, and the cells treated with simple medium were set as $100 \%$.

The tube formation assay of capillary-like structures inhibition was performed as described previously [36,38]. The $300 \mu \mathrm{L}$ matrix gel $(8.6 \mathrm{mg} / \mathrm{mL})$ was added to the 24 -well plates on ice and kept at $37^{\circ} \mathrm{C}$ for $30 \mathrm{~min}$ to polymerize into the membrane. Then HUVECs with the DPPNs, the aBev/DPPNs, or the aBev/cRGD-DPPNs in the pure medium were sown to the matrix gel. After $6 \mathrm{~h}$, images were taken under $20 \times$ inverted microscope and analyzed by Image J software.

\subsection{The Establishment of Rabbits CNV Model}

Male chinchilla rabbits weighting $2.5 \sim 3.0 \mathrm{~kg}$ used in this experiment were approved by the Ethical Committee for Care and Use of Laboratory Animals at Jilin University (Ethics approval number: SY201904012; Ethics approval date: 30 June 2019). All rabbits were cared for and treated in accordance with legal and institutional guidelines. The chinchilla rabbits with no abnormalities in the anterior segment and the fundus of the eyes were randomized into five groups: (1) Normal group: normal rabbits injected with $100 \mu \mathrm{L} 0.9 \%$ saline; (2) Control group: CNV rabbits injected with $100 \mu \mathrm{L}$ 0.9\% saline; (3) DPPNs group: CNV rabbits injected with $100 \mu \mathrm{L}$ DPPNs containing $0.1 \mathrm{mg}$ dexamethasone; (4) aBev/DPPNs group: CNV rabbits injected with $100 \mu \mathrm{L}$ aBev/DPPNs containing $0.1 \mathrm{mg}$ dexamethasone; (5) aBev/cRGD-DPPNs group: CNV rabbits injected with $100 \mu \mathrm{L}$ aBev/cRGD-DPPNs containing $0.1 \mathrm{mg}$ dexamethasone. The drug suspension was intravitreally injected to the right eyes with 27-needle 4 weeks after laser photocoagulation.

CNV model was induced by laser in chinchilla rabbits. Briefly, the healthy chinchilla rabbits were anesthetized with $10 \%$ chloral hydrate. Alcaine ${ }^{\circledR}$ and Mydriacyl ${ }^{\circledR}$ were following eye drops to the right eyes for conjunctival surface anesthesia and mydriasis. Then, the laser photocoagulation was carried out with an argon green laser with a wavelength of $532 \mathrm{~nm}$ (power $0.7 \mathrm{~W}$, exposure time $0.1 \mathrm{~s}$, 20 spots with diameter $50 \mu \mathrm{m}$, with the degree of bubble generation after photocoagulation, the bubble generation indicated that Bruce membrane rupture) (532 nm; Iris Radiation Systems, Povo, Italy). 


\subsection{In Vivo CNV Growth Inhibition Study}

The CNV growth inhibition study was studied by fluorescein fundus angiography (FFA). FFA was conducted 4 weeks after administration. The methods of anesthesia and mydriasis were the same as described in 3.8. The contrast agent of $10 \%$ fluorescein sodium $(0.2 \mathrm{~mL})$ and $0.9 \%$ saline $(1 \mathrm{~mL}) \mathrm{was}$ injected through the peripheral vein of the ear. FFA photo was recorded with high performance digital imaging system (Heidelberg HRA-II, Germany).

\subsection{In Vivo Immunohistochemistry and Histopathological Evaluation in RPE-Choroid}

Three rabbits in each group were killed by vein embolization at the ear edge after 4 weeks of vitreous administration. The right eyeball was removed and fixed in Verhoeff's solution for $24 \mathrm{~h}$ to enable a histological evaluation. The embedded eyeballs were sectioned continuously parallel to the sagittal axis of the optic nerve with a thickness of $5 \mu \mathrm{m}$. Slices of RPE-choroid were roasted in a $60{ }^{\circ} \mathrm{C}$ incubator for at least $1 \mathrm{~h}$. After dewaxing and hydration, the slices were filled with EDTA buffer solution and placed in a microwave oven for antigen repair. Subsequently, the slices were incubated with goat serum at $37^{\circ} \mathrm{C}$ for $10 \mathrm{~min}$, followed by a VEGF antibody at $37^{\circ} \mathrm{C}$ for $2 \mathrm{~h}$ and rinsed 3 times with TBS-T. The slices were subsequently blocked by goat serum at $37^{\circ} \mathrm{C}$ for $10 \mathrm{~min}$, followed by a biotinylated secondary antibody $\left(1 / 300\right.$, goat) at $37^{\circ} \mathrm{C}$ for $30 \mathrm{~min}$, and washed 3 times with TBS-T. The slices were subsequently sealed with Tween 20 and incubated with HRP-SA at $37^{\circ} \mathrm{C}$ for $30 \mathrm{~min}$. Finally, after TBS-T washing 3 times and TBS washing 5 times, the slices were stained with $3,3^{\prime}$-diaminobenzidine (DAB) and counterstained with hematoxylin. DAB tan color rendering was positive. The sections were imaged using a light microscope (Nikon Instruments Inc., Tokyo, Japan). The integrated optical density (IOD) of the positive staining area under $400 \times$ optical microscope was calculated by using Motic Images Advanced 3.2 software.

On the other hand, slices of RPE-choroid were stained with H\&E prior to histopathological observation of the retinas. A light microscope (Nikon Instruments Inc., Tokyo, Japan) at 200× magnification was used to examine the slices.

\subsection{In Vivo Downregulation of VEGF Protein}

VEGF protein amount in RPE-choroid was quantified using a Rabbit VEGF ELISA kit in accordance with manufacturer's instructions. ODs were measured at $450 \mathrm{~nm}$ (Multiskan Spectrum, Thermo, Waltham, MA, USA).

\subsection{Statistical Analysis}

All data are expressed as mean \pm standard deviations (SD). A one-way analysis of variance followed by the t-test was applied to all statistical comparisons. A $p$-value $<0.05$ was considered to indicate a significant difference.

\section{Conclusions}

The aBev/cRGD-DPPNs in this study showed size uniform and good biocompatible. In vitro uptake experiments showed that cRGD peptide could effectively promote the uptake of nanoparticles by ARPE-19. It can effectively inhibit the inhibition of angiogenesis in vivo and in vitro and has a good therapeutic effect on rabbit $\mathrm{CNV}$ in vivo.

In conclusion, the drug co-delivery nano carrier modified with cRGD peptide in this study can effectively deliver drugs to the site of AMD and exert effects, and the combination of dexamethasone and bevacizumab can better inhibit CNV. This study presents an ideal carrier, a new idea of combination medication, and targeted therapy for the treatment of AMD. 
Author Contributions: Conceptualization, Y.L.; data curation, J.L.; funding acquisition, Y.L.; investigation, F.X.; methodology, J.L.; resources, J.C.; software, L.L.; supervision, G.L.; validation, L.T.; visualization, F.X.; writing-original draft, J.L.; writing—review and editing, F.S. All authors have read and agreed to the published version of the manuscript.

Funding: This research received no external funding.

Conflicts of Interest: The authors declare no conflict of interest.

\section{References}

1. Bourne, R.R.A.; Flaxman, S.R.; Braithwaite, T.; Cicinelli, M.V.; Das, A.; Jonas, J.B.; Keeffe, J.; Kempen, J.H.; Leasher, J.; Limburg, H.; et al. Magnitude, emporal trends, and projections of the global prevalence of blindness and distance and near vision impairment: A systematic review and meta-analysis. Lancet Glob. Health 2017, 5, E888-E897. [CrossRef]

2. Ambati, J.; Fowler, B.J. Mechanisms of age-related macular degeneration. Neuron 2012, 75, 26-39. [CrossRef] [PubMed]

3. Tamura, A.; Ohashi, M.; Nishida, K.; Yui, N. Acid-induced intracellular dissociation of beta-cyclodextrinthreaded polyrotaxanes directed toward attenuating phototoxicity of bisretinoids through promoting excretion. Mol. Pharm. 2017, 14, 4714-4724. [CrossRef] [PubMed]

4. Kim, J.M.; Kang, S.W.; Son, D.Y.; Bae, K. Risk factors and clinical significance of prechoroidal cleft in neovascular age-related macular degeneration. Retin. J. Retin. Vitr. Dis. 2017, 37, 2047-2055. [CrossRef]

5. Ferris, F.L.; Fine, S.L.; Hyman, L. Age-related macular degeneration and blindness due to neovascular maculopathy. Arch. Ophthalmol. 1984, 102, 1640-1642. [CrossRef]

6. Angi, M.; Gibran, S.K.; Damato, B.E. Subfoveal choroidal neovascularization complicating 25-gauge trans-retinal choroidal tumor biopsy. Graef. Arch. Clin. Exp. 2008, 246, 1643-1645. [CrossRef]

7. Costagliola, C.; Campa, C.; Incorvaia, C.; Parmeggiani, F.; Menzione, M.; Della Corte, M.; Rinaldi, M.; Romano, M.; Semeraro, F. Verteporfin photodynamic therapy for subfoveal choroidal neovascularization in pathologic myopia: A 12-month retrospective review. Eur. J. Ophthalmol. 2008, 18, 955-959. [CrossRef]

8. Wang, M.; Munch, I.C.; Hasler, P.W.; Pruente, C.; Larsen, M. Central serous chorioretinopathy. Acta Ophthalmol. 2008, 86, 126-145. [CrossRef]

9. Caputo, M.; Zirpoli, H.; Di Benedetto, R.; De Nadai, K.; Tecce, M.F. Perspectives of choroidal neovascularization Therapy. Curr. Drug Targets 2011, 12, 234-242. [CrossRef]

10. Peyman, G.; Tsipursky, M.; Gohel, P.; Conway, M. Regression of peripapillary choroidal neovascularization after oscillatory transpupillary thermotherapy and anti-VEGF pharmacotherapy. Eur. J. Ophthalmol. 2011, 21, 162-172. [CrossRef]

11. Augustin, A.J.; Puls, S.; Offermann, I. Triple therapy for choroidal neovascularization due to agerelated macular degeneration-Verteporfin PDT, bevacimmab, and dexamethasone. Retin. J. Retin. Vitr. Dis. 2007, 27, 133-140.

12. Bakri, S.J.; Couch, S.M.; McCannel, C.A.; Edwards, A.O. Same-day triple therapy with photodynamic therapy, intravitreal dexamethasone, and bevacizumab in wet age-related macular degeneration. Retin. J. Retin. Vitr. Dis. 2009, 29, 573-578. [CrossRef] [PubMed]

13. Sivaprasad, S.; Patra, S.; DaCosta, J.; Adewoyin, T.; Shona, O.; Pearce, E.; Chong, N.V. A pilot study on the combination treatment of reduced-fluence photodynamic therapy, intravitreal ranibizumab, intravitreal dexamethasone and oral minocycline for neovascular age-related macular degeneration. Ophthalmologica 2011, 225, 200-206. [CrossRef]

14. Loyet, K.M.; Hass, P.E.; Sandoval, W.N.; Morando, A.; Liu, P.; Shatz, W.; Dickmann, L.; Kenrick, M.; Good, J.; Davancaze, T.; et al. In vivo stability profiles of anti-factor D molecules support long-acting delivery approaches. Mol. Pharm. 2019, 16, 86-95. [CrossRef]

15. Forte, R.; Cennamo, G.; Finelli, M.; Cesarano, I.; D'Amico, G.; de Crecchio, G.; Cennamo, G. Intravitreal triamcinolone, bevacizumab and pegaptanib for occult choroidal neovascularization. Acta Ophthalmol. 2010, 88, E305-E310. [CrossRef]

16. Ehmann, D.; Garcia, R. Triple therapy for neovascular age-related macular degeneration (verteporfin photodynamic therapy, intravitreal dexamethasone, and intravitreal bevacizumab). Can. J. Ophthalmol. 2010, 45, 36-40. [CrossRef] 
17. Segal, O.; Segal-Trivitz, Y.; Nemet, A.Y.; Cohen, P.; Geffen, N.; Mimouni, M. Anxiety levels and perceived pain intensity during intravitreal injections. Acta Ophthalmol. 2016, 94, 203-204. [CrossRef]

18. Fujita, N.; Fujita, S.; Ogata, N.; Matsuoka, M.; Okada, Y.; Kon, S.; Uede, T.; Saika, S. Endogenous osteopontin involvement in laser-induced choroidal neovascularization in mice. Investig. Ophthalmol. Vis. Sci. 2011, 52, 9310-9315. [CrossRef] [PubMed]

19. Hutton-Smith, L.A.; Gaffney, E.A.; Byrne, H.M.; Caruso, A.; Maini, P.K.; Mazer, N.A. Theoretical insights into the retinal dynamics of vascular endothelial growth factor in patients treated with ranibizumab, based on an ocular pharmacokinetic/pharmacodynamic model. Mol. Pharm. 2018, 15, 2770-2784. [CrossRef]

20. Ferrara, N. Vascular endothelial growth factor and age-related macular degeneration: From basic science to therapy. Nat. Med. 2010, 16, 1107-1111. [CrossRef]

21. Jager, R.D.; Mieler, W.F.; Miller, J.W. Medical progress: Age-related macular degeneration. N. Engl. J. Med. 2008, 358, 2606-2617. [CrossRef] [PubMed]

22. Ciulla, T.A.; Rosenfeld, P.J. Anti-vascular endothelial growth factor therapy for neovascular ocular diseases other than age-related macular degeneration. Curr. Opin. Ophthalmol. 2009, 20, 166-174. [CrossRef] [PubMed]

23. Hurwitz, H.; Fehrenbacher, L.; Novotny, W.; Cartwright, T.; Hainsworth, J.; Heim, W.; Berlin, J.; Baron, A.; Griffing, S.; Holmgren, E.; et al. Bevacizumab plus irinotecan, fluorouracil, and leucovorin for metastatic colorectal cancer. N. Engl. J. Med. 2004, 350, 2335-2342. [CrossRef] [PubMed]

24. Michels, S.; Rosenfeld, P.J.; Puliafito, C.A.; Marcus, E.N.; Venkatraman, A.S. Systemic bevacizumab (Avastin) therapy for neovascular age-related macular degeneration-Twelve-week results of an uncontrolled open-label clinical study. Ophthalmology 2005, 112, 1035-1047. [CrossRef] [PubMed]

25. Marneros, A.G.; Fan, J.; Yokoyama, Y.; Gerber, H.P.; Ferrara, N.; Crouch, R.K.; Olsen, B.R. Vascular endothelial growth factor expression in the retinal pigment epithelium is essential for choriocapillaris development and visual function. Am. J. Pathol. 2005, 167, 1451-1459. [CrossRef]

26. Penn, J.S.; Madan, A.; Caldwell, R.B.; Bartoli, M.; Caldwell, R.W.; Hartnett, M.E. Vascular endothelial growth factor in eye disease. Prog. Retin. Eye Res. 2008, 27, 331-371. [CrossRef]

27. Friedlander, M.; Theesfeld, C.L.; Sugita, M.; Fruttiger, M.; Thomas, M.A.; Chang, S.; Cheresh, D.A. Involvement of integrins alpha v beta 3 and alpha v beta 5 in ocular neovascular diseases. Proc. Natl. Acad. Sci. USA 1996, 93, 9764-9769. [CrossRef]

28. Hynes, R.O. Integrins: Bidirectional, allosteric signaling machines. Cell 2002, 110, 673-687. [CrossRef]

29. Dunehoo, A.L.; Anderson, M.; Majumdar, S.; Kobayashi, N.; Berkland, C.; Siahaan, T.J. Cell adhesion molecules for targeted drug delivery. J. Pharm. Sci. 2006, 95, 1856-1872. [CrossRef]

30. Ruoslahti, E. RGD and other recognition sequences for integrins. Ann. Rev. Cell Dev. Boil. 1996, 12, 697-715. [CrossRef]

31. Millard, M.; Odde, S.; Neamati, N. Integrin targeted therapeutics. Theranostics 2011, 1, 154-188. [CrossRef] [PubMed]

32. Mas-Moruno, C.; Rechenmacher, F.; Kessler, H. Cilengitide: The first anti-angiogenic small molecule drug candidate. Design, synthesis and clinical evaluation. AntiCancer Agent Med. Chem. 2010, 10, 753-768. [CrossRef]

33. Fangueiro, J.F.; Silva, A.M.; Garcia, M.L.; Souto, E.B. Current nanotechnology approaches for the treatment and management of diabetic retinopathy. Eur. J. Pharm. Biopharm. 2015, 95, 307-322. [CrossRef]

34. Sakurai, E.; Ozeki, H.; Kunou, N.; Ogura, Y. Effect of particle size of polymeric nanospheres on intravitreal kinetics. Ophthal. Res. 2001, 33, 31-36. [CrossRef] [PubMed]

35. Tian, S.; Cao, D.; Zou, H.; Bai, F.; Wang, Z.; Pan, S.; Feng, M. Endothelial cell-targeted pVEGF165 polyplex plays a pivotal role in inhibiting intimal thickening after vascular injury. Int. J. Nanomed. 2015, 10, 5751-5768. [CrossRef]

36. Liu, J.; Zhang, X.; Li, G.; Xu, F.; Li, S.; Teng, L.; Li, Y.; Sun, F. Anti-angiogenic activity of bevacizumab-bearing dexamethasone-loaded PLGA nanoparticles for potential intravitreal applications. Int. J. Nanomed. 2019, 14, 8819-8834. [CrossRef] [PubMed] 
37. Costa, R.; Carneiro, A.; Rocha, A.; Pirraco, A.; Falcao, M.; Vasques, L.; Soares, R. Bevacizumab and ranibizumab on microvascular endothelial cells: A comparative study. J. Cell. Biochem. 2009, 108, 1410-1417. [CrossRef] [PubMed]

38. Gong, C.; Deng, S.; Wu, Q.; Xiang, M.; Wei, X.; Li, L.; Gao, X.; Wang, B.; Sun, L.; Chen, Y.; et al. Improving antiangiogenesis and anti-tumor activity of curcumin by biodegradable polymeric micelles. Biomaterials 2013, 34, 1413-1432. [CrossRef] [PubMed]

Sample Availability: Samples of the compounds are not available from the authors.

Publisher's Note: MDPI stays neutral with regard to jurisdictional claims in published maps and institutional affiliations.

(C) 2020 by the authors. Licensee MDPI, Basel, Switzerland. This article is an open access article distributed under the terms and conditions of the Creative Commons Attribution (CC BY) license (http://creativecommons.org/licenses/by/4.0/). 